\title{
Expanding the mutation spectrum in 182 Spanish probands with craniosynostosis: identification and characterization of novel TCF12 variants
}

\author{
Beatriz Paumard-Hernández ${ }^{1}$, Julia Berges-Soria ${ }^{1}$, Eva Barroso ${ }^{1,2}$, Carlos I Rivera-Pedroza ${ }^{1}$, \\ Virginia Pérez-Carrizosa ${ }^{1}$, Sara Benito-Sanz ${ }^{1,2}$, Eva López-Messa ${ }^{1}$, Fernando Santos ${ }^{1,2}$, \\ Ignacio I García-Recuero ${ }^{3}$, Ana Romance ${ }^{3}$, María Juliana Ballesta-Martínez ${ }^{2,4}$, Vanesa López-González ${ }^{2,4}$, \\ Ángel Campos-Barros ${ }^{1,2}$, Jaime Cruz ${ }^{5}$, Encarna Guillén-Navarro ${ }^{2,4,6}$, Jaime Sánchez del Pozo ${ }^{5}$, \\ Pablo Lapunzina ${ }^{1,2}$, Sixto García-Miñaur ${ }^{1,2}$ and Karen E Heath ${ }^{\star, 1,2}$
}

Craniosynostosis, caused by the premature fusion of one or more of the cranial sutures, can be classified into non-syndromic or syndromic and by which sutures are affected. Clinical assignment is a difficult challenge due to the high phenotypic variability observed between syndromes. During routine diagnostics, we screened 182 Spanish craniosynostosis probands, implementing a four-tiered cascade screening of FGFR2, FGFR3, FGFR1, TWIST1 and EFNB1. A total of 43 variants, eight novel, were identified in $113(62 \%)$ patients: $104(92 \%)$ detected in level 1; eight (7\%) in level 2 and one (1\%) in level 3. We subsequently screened additional genes in the probands with no detected mutation: one duplication of the $\mathrm{IHH}$ regulatory region was identified in a patient with craniosynostosis Philadelphia type and five variants, four novel, were identified in the recently described TCF12, in probands with coronal or multisuture affectation. In the 19 Saethre-Chotzen syndrome (SCS) individuals in whom a variant was detected, $15(79 \%)$ carried a TWIST1 variant, whereas four $(21 \%)$ had a TCF12 variant. Thus, we propose that TCF12 screening should be included for TWIST1 negative SCS patients and in patients where the coronal suture is affected. In summary, a molecular diagnosis was obtained in a total of $119 / 182$ patients (65\%), allowing the correct craniosynostosis syndrome classification, aiding genetic counselling and in some cases provided a better planning on how and when surgical intervention should take place and, subsequently the appropriate clinical follow up. European Journal of Human Genetics (2015) 23, 907-914; doi:10.1038/ejhg.2014.205; published online 1 October 2014

\section{INTRODUCTION}

Craniosynostosis, the premature fusion of one or more cranial sutures, affects 1 in 2000-2500 children. ${ }^{1}$ It may occur due to genetic mutations or secondarily, due to mechanical, environmental and hormonal factors during pregnancy. The most frequently involved single suture is the sagittal suture followed by the coronal, metopic and lambdoid sutures, or multiple sutures. Craniosynostosis can be classified into non-syndromic (isolated) or syndromic when associated with other clinical features.

Three of the genes associated with craniosynostosis encode proteins belonging to the fibroblast growth factor receptor (FGFR) family; FGFR1 (MIM 136350), FGFR2 (MIM 176943) and FGFR3 (MIM 134934). The most commonly mutated gene is FGFR2, whereas the $\mathrm{p}$. Pro250Arg mutation in FGFR3 is the most frequent mutation, ${ }^{2}$ characteristic of the Muenke syndrome. ${ }^{3}$ EFNB1 (Ephrin B1, MIM 300035) encodes for a ligand, ephrin-B1, which binds to EphB receptors and has an important role in cell adhesion and the development and maintenance of the nervous system. ${ }^{4}$ Mutations in this gene, located on $\mathrm{Xq13.1}$, are associated with craniofrontonasal dysplasia. $^{5}$ The syndrome associated with mutations in EFNB1 is distinctive as females and male mosaics are more affected than males. ${ }^{6,7}$ Mutations in TWIST1 (MIM 601622) result in haploinsufficiency of the transcription factor TWIST1, and are associated with Saethre-Chotzen syndrome (SCS). In the early development of the coronal suture, TWIST1 is expressed in the sutural mesenchyme between the proliferating osteoblasts of the frontal and parietal bone edges, and overlapping with these two populations, consistent with roles in separating the two bone-forming tissues and with initiating and maintaining transcription of FGFR2. ${ }^{8}$

Recently, mutations in another gene, TCF12 (MIM 600480), have been identified in patients with coronal synostosis, many of which were initially referred with SCS, and in whom no TWIST1 mutation had been identified. ${ }^{9}$ TCF12 encodes transcription factor 12 (TCF12), a member of the basic helix-loop-helix (bHLH) E-protein family. It is expressed in many tissues, among them bone, skeletal muscle, thymus, $\mathrm{B}-$ and $\mathrm{T}$ cells, and may participate in regulating lineage-specific gene expression through the formation of heterodimers with other bHLH E-proteins, such as TWIST1. In a study of 347 patients with craniosynostosis, 36 TCF12 mutations were detected, the majority of which resulted in exon splicing or altered the reading frame. ${ }^{9}$

${ }^{1}$ Institute of Medical and Molecular Genetics (INGEMM), Hospital Universitario La Paz, Universidad Autónoma de Madrid, IdiPAZ, Madrid, Spain; ${ }^{2}$ Centro de Investigación Biomédica en Enfermedades Raras (CIBERER), Instituto Carlos III, Madrid, Spain; ${ }^{3}$ Maxilofacial Surgery Unit, Hospital 12 de Octubre, Madrid, Spain; ${ }^{4}$ Medical Genetics Unit, Department of Pediatrics, Hospital Clínico Universitario Virgen de la Arrixaca, Madrid, Spain; ${ }^{5}$ Dysmorphology Unit, Hospital Universitario Doce de Octubre, Madrid, Spain; ${ }^{6}$ Cátedra de Genética Médica. UCAM-Universidad Católica San Antonio de Murcia, Madrid, Spain

*Correspondence: Dr KE Heath, Instituto de Genética Médica y Molecular (INGEMM), Hospital Universitario La Paz, Po Castellana 261 , 28046 Madrid, Spain. Tel: +34 912071010 Ext.269; Fax: +34 91207 1040; E-mail: karen.heath@salud.madrid.org

Received 23 April 2014; revised 26 August 2014; accepted 29 August 2014; published online 1 October 2014 
More recently, a patient with coronal craniosynostosis and intellectual disability was found to have a complex balanced maternal chromosomal rearrangement combining the reciprocal translocation of the region and an insertion which resulted in the deletion of TCF12. ${ }^{10}$

Another gene recently implicated in craniosynostosis, ERF (MIM 611888), encodes for the transcription factor ERF (Ets2 repressor factor). Mutations in ERF were identified in 12/411 patients with a complex form of craniosynostosis. ${ }^{11}$

Phenotypic variability in craniosynostosis makes the clinical diagnosis difficult; thus, genetic testing can support or aid the clinical diagnosis and improve genetic counselling in these families. Evidence also suggests that molecular diagnosis can help to define the treatment or surgery necessary in the short to medium term and predict the clinical evolution. ${ }^{12,13}$ Patients with an identified TWIST1 mutation have a high rate of reoperation due to intracranial hypertension, ${ }^{12}$ whereas mutations in TCF12 do not. ${ }^{9}$ Thus, the knowledge of the genetic mutation permits greater monitoring of the intracranial hypertension in these patients. Likewise, other studies have confirmed that the frequency of transcranial surgery performed to reduce intracranial pressure is much higher in patients with Muenke syndrome. ${ }^{14}$ It is also important to check for hearing loss in patients with Muenke, as it has been reported that $20 \%$ require hearing aids. ${ }^{15}$

In 2010, Wilkie et al, ${ }^{2}$ presented a prospective cohort of 326 patients with craniosynostosis, where the molecular aetiology was identified in $84,86 \%$ of the mutations were monogenic alterations, whereas $14 \%$ were chromosomal abnormalities. The employed molecular screening strategy was a two-tier cascade screening protocol, including the analysis of various exons of FGFR1, FGFR2, FGFR3, TWIST1 and EFNB1 at each level, depending on the incidence of their mutations in the population. The incidence of mutations in FGFRs were FGFR2 (32\%), FGFR3 (25\%), TWIST1 (19\%) and EFNB1 (7\%). ${ }^{2}$ This study formed the basis of our work, which aimed to improve the genetic diagnosis of craniosynostosis in the Spanish population, but also resulted in the screening of the entire coding sequence and intron: exon boundaries of these genes and mutation analysis of TCF12 and ERF.

\section{MATERIALS AND METHODS}

\section{Cohort}

All participants provided written informed consent for the performed studies and ethical approval was obtained from the respective participating institutions. Clinical details were obtained for all patients recruited into the study. The cohort was composed of 182 probands with a clinical diagnosis of craniosynostosis and 89 family members. All samples were reported to have a normal G-banding karyotype. Genomic DNA was isolated from whole blood (Blood kit; Qiagen (Valencia, CA, USA) or Chemagic DNA extraction special, Perkin Elmer Chemagen (Perkin Elmer Technologie GmbH, Baesweiler, Germany)).

\section{Molecular analysis}

The screening protocol consisted of four levels and included the analysis of FGFR1 exon 7 (NM_015850.3 NG_007729.1), FGFR2 exons 2-18 (NM_ 000141.4, NG_012449.1, FGFR2 exons 7 and 8 are alternatively known as exon IIIa and IIIc, respectively ${ }^{16}$ ), FGFR3 exons 2-18 (NM_000142.4 NG_0126321), TWIST1 exon 1 (NM_000474.3, NG_008114.1) and EFNB1 exons 1-5 (NM_004429.4, NG_008887.1) (Supplementary Figure 1), TCF12 exons 2-20 (NM_207037.1) and ERF exons 1-4 (NM_004429.2). Oligonucleotides were designed for all exons and intron-exon boundaries of the genes of interest with the help of OLIGO V6 software and SNPCheck V3 (https://secure. ngrl.org.uk/SNPCheck/snpcheck.htm). The genes were screened for mutations by high-resolution melting (HRM) analysis using the HR96 Light Scanner (BioFire Diagnostics, Salt Lake City, UT, USA). The sensitivity of HRM in our laboratory is $99 \%$. As EFNB1 is located on the X-chromosome and there is a
}

high incidence of mosaicism, ${ }^{6}$ all samples were analysed for this gene by HRM and sequencing. The sequences and PCR conditions for FGFR1, FGFR2, FGFR3, TWIST1 and EFNB1 are shown in Supplementary Table 1, for TCF12 (Supplementary Table 2) and for ERF (Supplementary Table 3). Any abnormal HRM profile was subsequently sequenced using the Big-Dye Terminator v3.1 kit (Applied Biosystems, Foster City, CA, USA) on an ABI3730XL DNA Sequencer (Applied Biosystems, Carlsbad, CA, USA). The results were analysed using Sequencher v5.10 (Gene Codes Corporation, Ann Arbour, MI, USA). MLPA (Multiplex Ligation-dependant Probe Amplification) P080B1 (MRC Holland, The Netherlands) was used to detect copy number alterations.

\section{Pathogenicity assessment}

The pathogenicity of the detected alterations was assessed by determining: (1) If the mutation had been previously reported; (2) If not, the variations were assessed using the programs Ensembl and NCBI dbSNP to determine if the alteration has been described as a polymorphism. Allelic frequencies were obtained using NHLBI Exome Sequencing Project (ESP) Exome Variant Server (EVS) and the 1000 genomes, and where necessary in 300 Spanish healthy controls; (3) Amino acid conservation analysis; (4) Prediction of the function and pathogenicity using the bioinformatics package, Alamut 2.4.1 which includes PolyPhen, SIFT, MutationTaster and various splicing predictor tools (Human Splicing Finder (HSF), MaxEntScan, Splice Site Finder (SSF), GeneSplicer, Splice Site Prediction by Neural Network (NNsplice); (5) Functional analysis of splicing variants using minigene assays.

All variants and individuals have been submitted to the gene variant database at www.LOVD.nl/CAV3.

\section{Minigenes assay for TCF12 splicing variants}

Minigene constructions based on the pSPL3 exon trapping vector (kind donation from Dr Belen Perez) were used to determine those variants predicted to affect splicing actually affected exon splicing. The minigene assay was based on the protocol as previously described. ${ }^{17}$ For these putative splicing variants, the exon and intronic flanking sequences were amplified from the patient's DNA, using primers detailed in Supplementary Table 4. Minigene constructions were confirmed by bidirectional sequencing.

\section{RESULTS}

In this study, the cascade screening protocol permitted the identification of genetic variants in a total of $113 / 182$ (62.1\%) patients (Table 1): 67 FGFR2, 29 FGFR3, 15 TWIST1 and two EFNB1 variants (Figure 1). The variant was identified in 104/182 patients in level 1 (57.1\%), eight $(4.4 \%)$ in level 2 , one in level $3(0.55 \%)$, whereas no mutations were identified in level 4 . A total of 43 different mutations, eight of which were novel, were identified, four in FGFR2, three in TWIST1 and one in FGFR3. Predictive analysis of the pathogenicity of the novel variants was undertaken (Table 1). Parental analysis was possible in a total of 34 probands: 19 were de novo events, whereas 15 were inherited from a clinically affected parent.

We also analysed the recently reported genes TCF12 and ERF. Five variants were identified in TCF12 (Table 2), four were novel whereas one, p. (Ser281*), was previously described in two families. ${ }^{9}$ The clinical characteristics of these five probands and family members are shown in Table 3. Cosegregation analysis was possible in four of the families (Supplementary Figure 2). The c.826-2A $>$ G splicing mutation and the p. (Ser281*) mutations were shown to have arisen as de novo events or due to germline mosaicism (Supplementary Figure 2) whilst the c. $1144 \mathrm{C}>\mathrm{T}\left(\mathrm{p} .\left(\mathrm{G} \ln 382^{\star}\right)\right)$ and $\mathrm{p}$.(Leu507Arg) variants were present in affected and normal individuals. Two non-synonymous variants and a variant at c. $-1 G>A$ were identified but were subsequently shown to be non-pathogenic (Supplementary Table 5).

In silico prediction analysis was undertaken for the novel TCF12 variants (Table 2). The intron 10 splicing mutation, c.826-2A $>\mathrm{G}$, affects one of the two highly conserved nucleotides implicated in 
Table 1 Description of the 113 mutations (43 different) identified in FGFR2, FGFR3, TWIST1 and EFNB1 for each syndrome within each level and the predictive pathogenicity

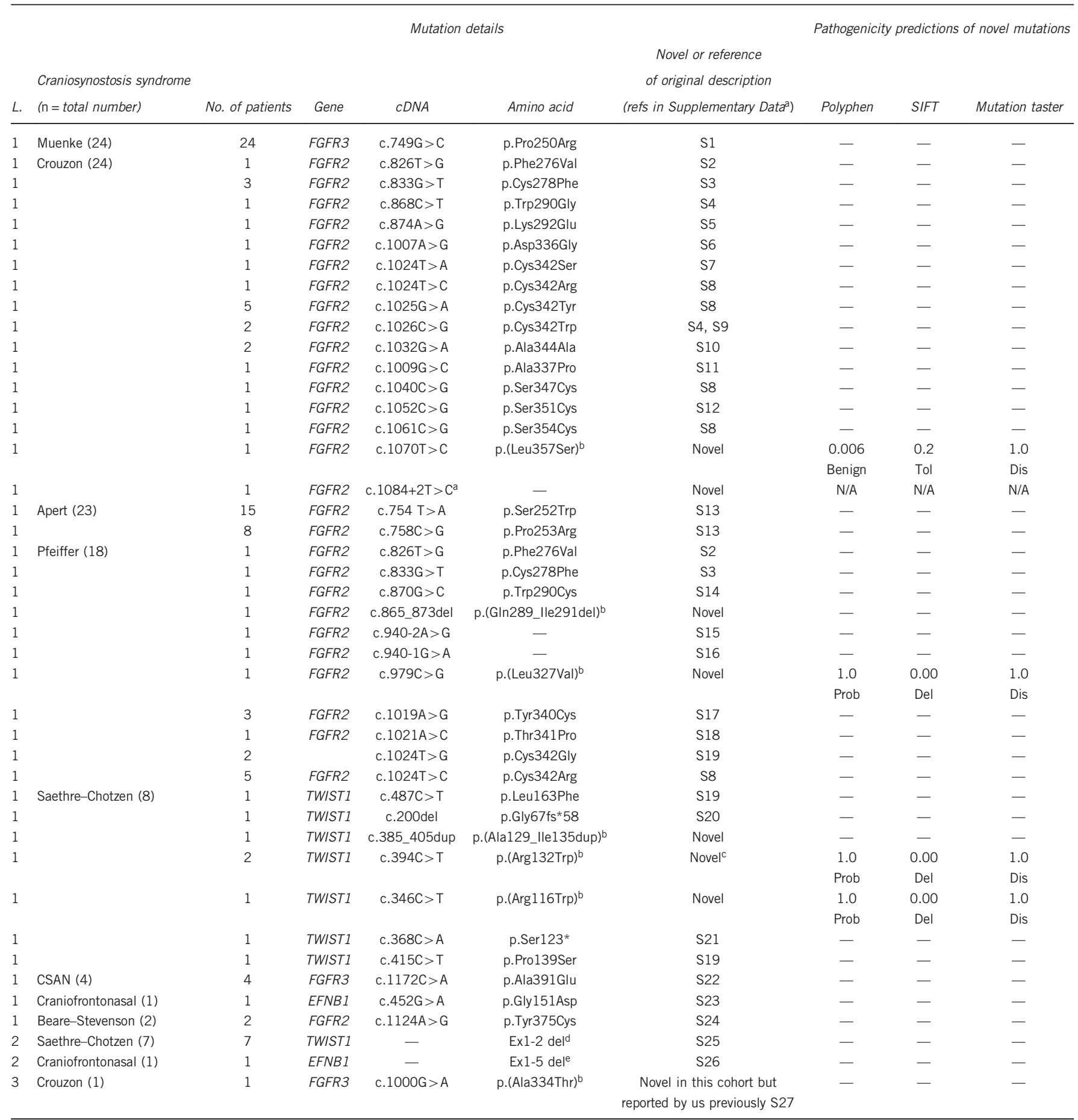

Abbreviations: CSAN, Crouzon syndrome with acanthosis nigricans; FGFR, fibroblast growth factor receptor; L., mutation screening level; N/A, not applicable.

aReferences to the first citation of the mutation are indicated in Supplementary Data (S1, S2 and so on).

bThe predicted effect on the protein of the novel variants is indicated. PolyPhen: Prob - probably disease causing; Pos - possibly disease causing; Benign - predicted to be non-disease causing. SIFT: Del-deleterious, that is, disease causing, Tol - tolerated, that is, non-disease causing. MutationTaster: Dis - disease causing. N/A not applicable as it affects splicing.

CHaplotype analysis using microsatellite markers D7S2559, D7S2495 and TWI-CA in the two families suggested a common ancestor. All variants/individuals have been submitted to the LOVD database at www.LOVD.nl/CAV3.

dSix cases had a deletion of exons 1 and 2 (Chr7.hg19:g.(? 19156729) (19155420 ?)del or c.316-? *377+?del) detected by a deletion of MLPA probes L2886, L2364, L0722 and L1592 (P080A1) or L16137, L02364, L16216 and L01598 (P080B1). One had a larger deletion (Chr7.hg19:g.(?_19738208)_(19155420_?)del) which included the upstream pseudogene TWISTNB exon 4 (L16629) but this patient did not have intellectual disabilities.

eThe two CFNS cases were females. Both had deletions of exons 1-5 inclusive (ChrX.hg19:g.(?_68049606)_(68060248_?)del) detected by a deletion of MLPA probes L16740, L16124, L16121, L16113 and L16135 (P080B1). 


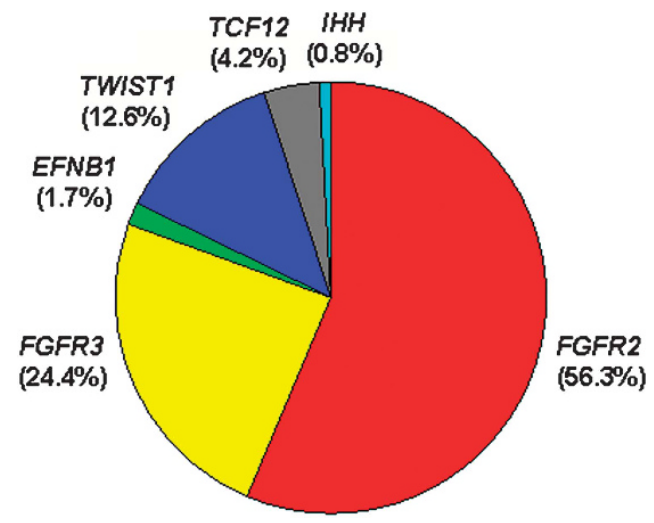

Figure 1 Pie-chart representation of the frequency of mutations identified in the studied genes. The particular mutations identified are documented in Table 1 , plus the duplication of the $I H H$ regulatory elements. A total of 27 FGFR2 mutations were identified in 67 patients. Three FGFR3 mutations were identified in 29 patients, 24 with the p.Pro250Arg mutation, four with the p.Ala391Asn and one with a novel p.Ala334Thr mutation. Two EFNB1 mutations were identified in two patients, eight TWIST1 mutations in 15 probands and five TCF12 mutations in five probands.

splicing. Predictive splicing tools predicted that this TCF12 mutation could disrupt the canonical splice acceptor site and shift the $3^{\prime}$ acceptor splice site $2 \mathrm{bp}$ downstream of the canonical one. A minigene assay showed that indeed the intronic substitution affected splicing of exon 11 but unexpectedly, two splicing products were observed for the mutant, c.826-2G $>$ A. One was as predicted, resulting in c.826_827del (p.Asn276LeuFs ${ }^{\star} 61$ ), whereas the second splicing product corresponded to the complete deletion of exon 11 (Figure 2).

In addition, one proband with a clinical diagnosis of craniosynostosis Philadelphia type had an $\sim 31 \mathrm{~kb}$ duplication of the $I H H$ regulatory sequences (Chr2(GRCh36):g.219658383_219689640dup) (Barroso et al, manuscript in preparation). Thus, a variant was identified in $119 / 182$ patients.

\section{DISCUSSION}

A cascade system for the genetic diagnosis of craniosynostosis was assessed in a total of 182 probands with craniosynostosis. Mutations were identified in a total of $113(62.1 \%)$ of patients. As in a similar UK study, ${ }^{2}$ the most frequent mutation was the characteristic Muenke syndrome mutation, FGFR3 p.Pro250Arg, which was detected in a total of 24 patients (13.2\% of cohort), although some what lower than the $24 \%$ detected in the UK. Although the mutated gene frequency order was the same, the frequency of FGFR2 mutations was significantly different to the UK study (32\%), but similar to that detected in a cohort of 630 Australian craniosynostosis patients $(62 \%) .{ }^{13}$ This is principally due to the high incidence of Apert and Pfeiffer cases in these cohorts compared to the UK cohort.

In the 113 patients with a confirmed molecular diagnosis, the genetic diagnosis confirmed the prior clinical diagnosis in 93 cases (82\%). More importantly, the molecular diagnosis permitted a correction of the assigned craniosynostosis syndrome in a total of 20 patients (18\%, Supplementary Table 6). This was demonstrated with the group of 48 patients that were referred with a clinical diagnosis of Crouzon syndrome, 21 (44\%) were genetically confirmed whilst 10 patients had been clinically misclassified and were found to have the FGFR3 p.P250R mutation, characteristic of Muenke syndrome. Another example was demonstrated by the 36 cases referred with Muenke syndrome. Only $11(31 \%)$ of them actually had the characteristic FGFR3 mutation. However, this mutation was identified in an additional 13 patients who were referred for other craniosynostosis syndromes, such as Crouzon and Pfeiffer syndrome. Some of these misclassifications may be due to referrals from non-specialised centres; however, others were due to clinical overlap. For example, the p.Pro250Arg mutation was detected in a patient with bicoronal craniosynostosis, mild cutaneous syndactyly and malformation of both thumbs and big toes, more often associated with SCS or Pfeiffer syndrome and rarely in Muenke syndrome. These data suggest the need to screen this mutation in all patients, regardless of the referred clinical diagnosis.

In level 1, two prenatal craniosynostosis cases were genetically diagnosed with the FGFR3 p.Tyr375Cys mutation, characteristic of Beare-Stevenson syndrome (BSS). In 21 BSS cases reported in the literature, ${ }^{18}$ FGFR2 mutations have been identified in 16 of these, 14 with the p.Tyr375Cys mutation and the remaining two with p. Ser372Cys. ${ }^{19}$ The prognosis of FGFR2-related BSS is poor. Of the 13 BSS patients in whom a FGFR2 mutation was found and for whom age of death was reported, $40 \%(n=5)$ died within 1 month. Only 4/13 BSS patients were still alive after 1 year, with the oldest reported so far being 4 years old. ${ }^{20}$

In some cases, the characteristic clinical feature additional to the craniosynostosis, had not yet developed thus, not permitting the correct clinical assignment, as is the case with the presentation of acanthosis nigricans in Crouzon syndrome with acanthosis nigricans (CSAN). All four cases were referred as neonates. The identification of the FGFR3 p.Ala391Asn mutation permitted the correct syndrome assignment and the anticipation of additional complications and thus, appropriate clinical monitorization. Two of the cases had been previously reported: one presenting with craniosynostosis and developing acantosis nigricans at 21 months of age, the youngest reported to date, whereas the other case had craniosynostosis, acanthosis nigricans from the age of 4 years. ${ }^{21}$ The other two are recent referrals that represent the clinical variability of this syndrome: a 5-month old girl referred for Crouzon syndrome but to date, presented with no other manifestations, whereas, in contrast, the 3-month old girl presented with more severe physical manifestations and indicative of CSAN, such as choanal stenosis and Chiari type I malformation.

In contrast, the clinical diagnosis of the Apert syndrome is far easier, thus permitting a correct clinical classification. This is shown in our cohort where the two characteristic Apert FGFR2 mutations, p. Ser252Trp and p.Pro253Arg, were detected in 23/27 (85\%) patients. The remaining four were referred as possibly Apert syndrome and are thus likely to have another type of craniosynostosis, as these two FGFR2 mutations account for $>98 \%$ of Apert cases. To date, only four other FGFR2 mutations in five patients have been reported. ${ }^{22-26}$ The entire coding region of FGFR2, and deletions or Alu events in FGFR2 have been excluded.

FGFR2 exons 7 and 8 (alternatively known as exon 8 (IIIa) and exon 10 (IIIc) ${ }^{16}$ ) are the two regions where the majority of mutations were identified in Apert, Crouzon and Pfeiffer syndromes. This is in accordance with the data observed in previous studies. ${ }^{13,27}$ Therefore, these two regions, along with FGFR3 exons 7 and 9 (otherwise known as exon 7 (IIIc) and exon $10\left(\mathrm{TM}^{16}\right)$ ) should be included in the first level of genetic analysis. A total of eight deletions were detected, seven TWIST1 deletions and one EFNB1 deletion, in level 2. Due to this high incidence, we recommend that deletions and duplications of these genes should be rather carried out in level 1 for SCS and CFNS referrals.

Level 3 analyses only permitted the genetic diagnosis of one proband, clinically diagnosed with a mild Crouzon-like 
Table 2 Summary of the five TCF12 variants

\begin{tabular}{|c|c|c|c|c|c|c|c|c|c|c|c|}
\hline Proband & $c D N A^{a}$ & $\begin{array}{l}\text { Exon/ } \\
\text { intron }\end{array}$ & Amino acid & Domain & $\begin{array}{l}\text { Cosegregates } \\
\text { or de novo and } \\
\text { penetrance }\end{array}$ & $\begin{array}{l}\text { Novel or } \\
\text { reported }\end{array}$ & $\begin{array}{l}\text { Amino acid } \\
\text { conserved }\end{array}$ & $\begin{array}{c}\text { Mutation } \\
\text { taster }\end{array}$ & Polyphen & SIFT & $\begin{array}{l}\text { Splicing prediction } \\
\text { tools }{ }^{b} \text { or minigene } \\
\text { splicing assay }\end{array}$ \\
\hline 1 & c.596dup & Ex 9 & $\begin{array}{l}\text { p. } \\
(\text { Asn200Lysfs*4) }\end{array}$ & - & ND & Novel & - & - & - & - & - \\
\hline 2 & c. $842 C>G$ & Ex 11 & p.(Ser281*) & - & De novo & Ref. 9 & $\begin{array}{c}\text { Highly } \\
\text { conserved }\end{array}$ & - & - & - & - \\
\hline 3 & c. $826-2 A>G$ & $\begin{array}{l}\text { Int } \\
10\end{array}$ & - & $\begin{array}{l}\text { Activation } \\
\text { domain } 2\end{array}$ & De novo & Novel & - & - & - & - & $\begin{array}{l}\text { Predicted to ablate } \\
\text { splicing acceptor site. } \\
\text { Minigene assay revealed } \\
\text { the creation of two aber- } \\
\text { rant splicing products } \\
\text { (Figure 2). }\end{array}$ \\
\hline 4 & c. $1144 \mathrm{C}>\mathrm{T}$ & Ex 14 & p. $\left(G \ln 382^{*}\right)$ & $\begin{array}{l}\text { Activation } \\
\text { domain } 2\end{array}$ & $\begin{array}{l}\text { Cosegregation and } \\
\text { incomplete pene- } \\
\text { trance (unaffected } \\
\text { cousin) }\end{array}$ & Novel & $\begin{array}{c}\text { Highly } \\
\text { conserved }\end{array}$ & - & - & - & - \\
\hline 5 & c. $1520 T>\mathrm{G}^{\mathrm{c}}$ & Ex 17 & p.(Leu507Arg) & $\begin{array}{l}\text { Activation } \\
\text { domain } 2\end{array}$ & $\begin{array}{l}\text { Incomplete pene- } \\
\text { trance (unaffected } \\
\text { father) }\end{array}$ & $\begin{array}{l}\text { rs36060670 } \\
\text { (EVS:11/2184 } \\
\text { European } \\
\text { Americans) }\end{array}$ & $\begin{array}{l}\text { Moderately } \\
\text { conserved }\end{array}$ & $\begin{array}{l}1.0 \\
\text { Dis }\end{array}$ & $\begin{array}{c}0.4 \\
\text { Benign }\end{array}$ & $\begin{array}{c}0.01 \\
\text { Del }\end{array}$ & - \\
\hline
\end{tabular}

Abbreviations: EVS, Exon variant Server; ND, not determined.

The predictive pathogenicity is indicated for the novel alterations.

a TCF12 transcript NM_207037.1.

${ }^{\mathrm{b}}$ Five splicing tools available in Alamut V2.0, SpliceSite Finder-like, MaxEntScan, NNSPlice, GeneSplicer and Human Splicing Factor. Patients and variants have been submitted to the gene variant database at www.LOVD.nl/CAV3.

CPathogenicity uncertain until functional analysis is undertaken. Predictive pathogenicity tools: PolyPhen: Benign—predicted to be non-disease causing. SIFT: Del—deleterious. MutationTaster:

Dis-disease causing.

craniosynostosis and that was found to have a novel FGFR3 mutation, p.Ala334Thr, which we previously reported. ${ }^{28}$ No mutation was detected in level 4 suggesting that these regions of the genes are not a common cause of craniosynostosis. Besides the cascade screening, we searched for a specific mutation in one family who was referred with the rare craniosynostosis, Philadelphia type (MIM 185900). The identification of a duplication of the upstream $I H H$ enhancers confirmed this diagnosis. ${ }^{29}$

During the course of the project, two novel craniosynostosis genes were identified; TCF12 (Sharma et al ${ }^{9}$ ) and ERF. ${ }^{11}$ We subsequently screened both genes in the 72 patients, which remained genetically undiagnosed after the cascade screening. Five patients were found to have a TCF12 variant, accounting for $2.7 \%$ of the total cohort. Of the five TCF12 variants, one had been previously reported and four were novel, three clearly pathogenic, and one of unknown significance. We subsequently assessed the pathogenicity of the novel variants using both prediction analyses and minigene assays where necessary. The de novo splice site alteration, TCF12 variant c.826-2A $>$ G, identified in proband 3, affects one of the two highly conserved splice site nucleotides in the intron 10 splice site acceptor. A minigene assay confirmed experimentally the predicted splicing effects of the TCF12 variant c.826-2A $>$ G: the mutation had a dual effect on splicing, leading to exon 11 skipping and also generating an alternative transcript with the recognition of a cryptic acceptor site (c.826_827del), leading to the premature termination (p.Ser276Leufs $\left.{ }^{\star} 61\right)$. In both events, the mutant transcripts are predicted to be non-functional as they either affect the activation domain 2 of TCF12 or result in a prematurely truncated protein, which may be degraded by nonsense mediated decay.

The c.596dup (p. (Asn200Lysfs $\left.\left.{ }^{\star} 4\right)\right)$ and c.1144C $>$ T (p. $($ Gln382*)) variants observed in probands 1 and 4 , respectively, are predicted to result in the premature truncation of the protein. Cosegregation analysis in the family of proband 4 revealed that all members affected with SCS carried the TCF12 mutation, but two unaffected individuals were shown to be carriers, thus incomplete penetrance was observed. Further examination of one of these individuals was possible (III.5), in whom mild cranial deformity was subsequently observed. This could represent a minimal expression of SCS, but unfortunately no MRI was undertaken to enable a more detailed judgment.

The only TCF12 missense variant, p.(Leu507Arg), identified in proband 5 and the unaffected father, is of unknown significance but is predicted to be pathogenic. The variant affects a highly conserved nucleotide and amino acid. This substitution was absent in 400 Spanish healthy controls but present in $11 / 2184 \quad(\mathrm{MAF}=0.005)$ European Americans in the EVS database. As the EVS population has not been excluded for craniosynostosis and incomplete penetrance is a common phenomenon, functional analysis will be required to definitely determine the pathogenicity of this variant.

Mutations in TCF12 have been reported predominantly in patients with unilateral or bilateral coronal craniosynostosis. In the previously studied cohort, 14/38 mutations were identified in patients referred for SCS in whom no TWIST1 mutation had been identified. ${ }^{9}$ Four of our five patients were initially referred for SCS, whereas the fifth was referred for the Muenke syndrome. In the 19 SCS individuals in whom a TWIST1 or TCF12 variant has been detected, 15 patients $(79 \%)$ carried a TWIST1 variant, whereas four (21\%) had a TCF12 variant. The other case allowed reassignment of the craniosynostosis syndrome, from the Muenke syndrome to TCF12-related coronal craniosynostosis. We were able to clinically evaluate nine individuals from the five families with TCF12 variants (Table 3), although not all details were available for each individual. The affected sutures included coronal unilateral (5/7) or multiple sutures including the coronal $(2 / 7)$. 


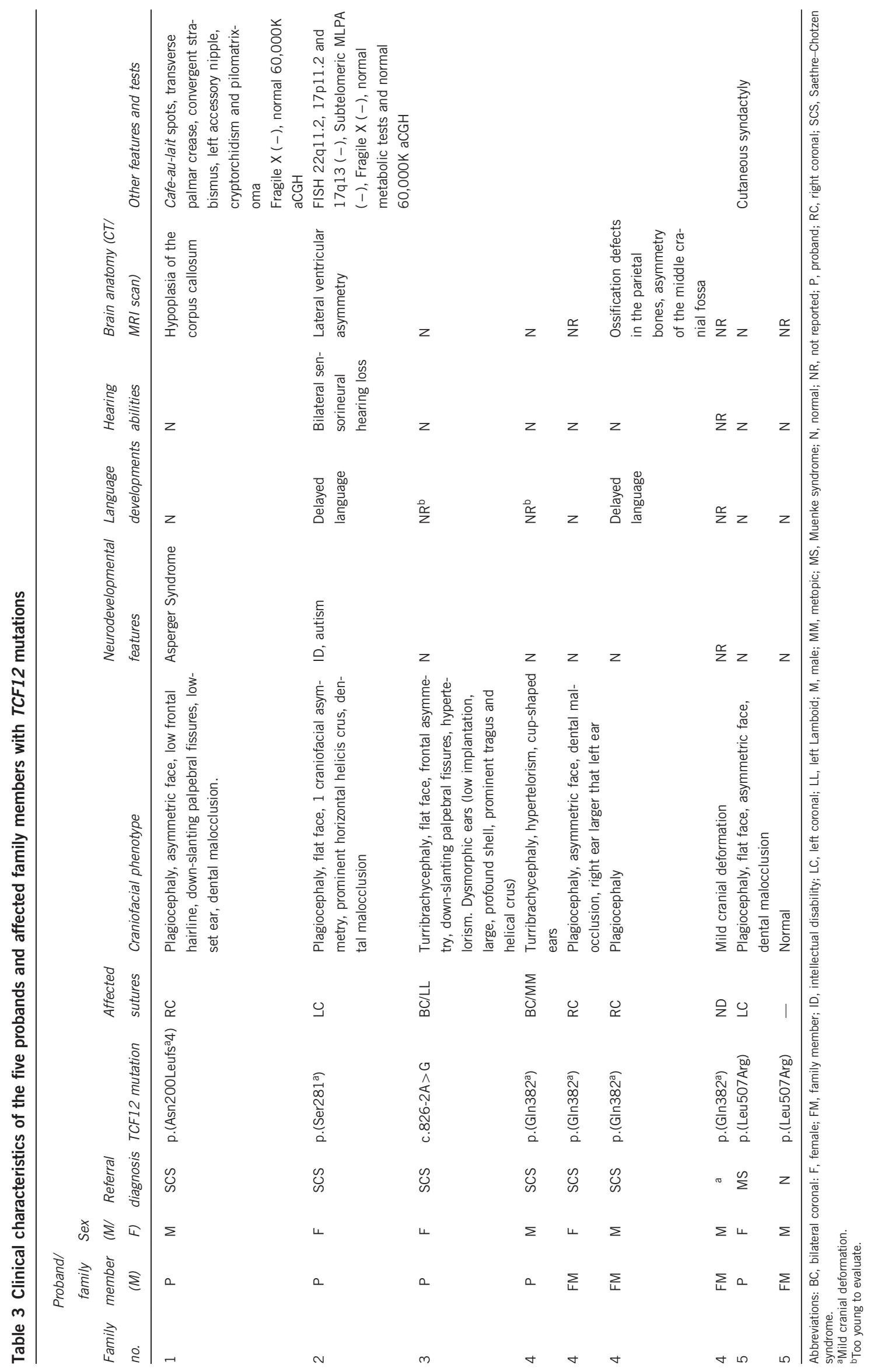


a

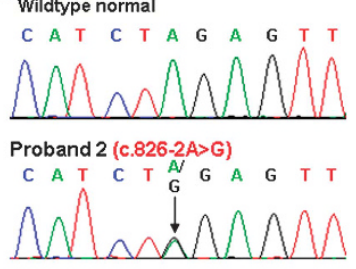

b
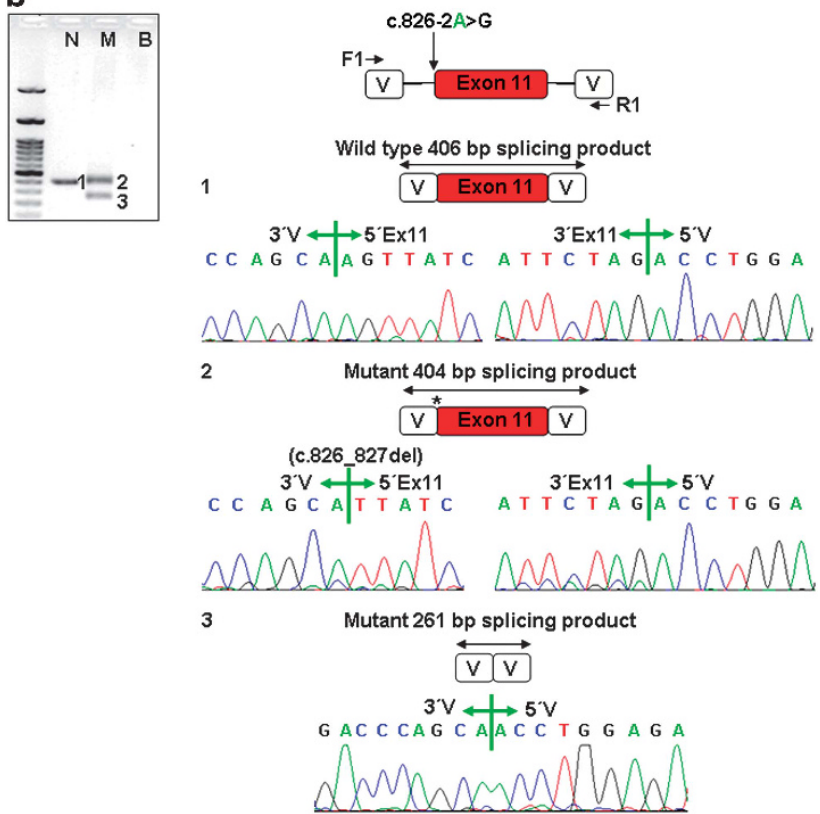

Figure 2 Genetic and functional analysis of the c.826-2A $>$ G TCF12 mutation, identified in proband 3. (a) Sequence chromatogram of the c.826$2 A>G$ mutation of TCF12. (b) In the left panel, gel electrophoresis of the splicing products generated from the amplification of the wild-type and mutant cDNA isolated from transfected HEK293 cells, using the two vector primers ( $F 1$ and $R 1$, sequences available in methods), comparing the mutant c.826G with the wild-type c.826A alleles. $\mathrm{N}$, normal wild-type c.826A PCR product; M, mutant c.826-2G PCR product and B, PCR blank. In the right panel, a schematic representation and chromatograms of the splicing products, visualized in the agarose gel. The sequences show the two junctions, the $3^{\prime}$ end of the vector:exon 11 and the exon 11: and the $5^{\prime}$ of the vector. Band 1 (406 bp) corresponds to the wild-type exon 11 transcript, band $2(404 \mathrm{bp})$ corresponds to one of the two aberrant transcripts, where the splicing machinery utilizes a different splice donor, resulting in the deletion of the first $2 \mathrm{bp}$ of exon 11, c.826_827del and band 3 (261 bp) corresponds to the second aberrant transcript which arises due to exon 11 skipping thus only vector sequence is observed. $\mathrm{V}$, vector.

The MRI of proband 4 with the p.(Ser382*) mutation is shown in Supplementary Figure 3. The phenotypes of five of the seven cases were less specific: facial asymmetry (5/7), flat-facies (3/7) and ear malformations (5/7). Bilateral sensorineural hearing loss was only reported in one of the seven individuals. Delayed language was reported in two individuals, one of whom had hearing loss, whereas two individuals were too young to evaluate.

The atypical case, proband 1, was a severely affected boy with the de novo p.(Ser281*) mutation, who presented unilateral coronal synostosis, sensorineural bilateral hearing loss, mental retardation, autism and language skills delay and asymmetric lateral ventricles. Microdeletion syndromes, metabolic errors and Fragile X syndrome were not detected (Table 3), thus decreasing the likelihood of a concurrent cause of developmental delay. This mutation has been previously reported in three male cases, two related, all of whom only had the coronal suture (uni or bilateral) affected. ${ }^{9}$ Neurodevelopment was normal in two of the cases, whereas mild learning disability was reported in the third case. Language skills were normal. Other clinical characteristics included cornea abnormalities in one patient and low frontal hairline and incomplete descent of testes in another. In the brain CT/MRI scan, one patient had a small mass near the pineal gland and another patient had mild ventriculomegaly. Thus, no genotype:phenotype correlation was observed between the p.(Ser281*) affected individuals.

Proband 2 with the de novo mutation p.(Asn200Lysfs $\left.{ }^{\star} 4\right)$, has intellectual disability, malformation of the corpus callosum, facial dysmorphic features, ophthalmological malformation, transverse palmar crease and cryptorchidism, also described in a patient by Sharma et al. ${ }^{9}$ In the two familial cases of TCF12 mutations, probands 4 and 5, incomplete penetrance was observed in $3 / 5(60 \%)$ tested family members, with one case showing mild cranial deformity, but no other symptoms. This is in line with the data reported by Sharma et al. ${ }^{9}$

Thus, TCF12 should be incorporated for the screening of TWIST1 negative SCS cases and FGFR3 p.Pro250Arg Muenke negative cases. In contrast to the original report of a significant number of $E R F$ mutations in multiple-suture craniosynostosis, we failed to detect a mutation in this gene. This study suggests that the frequency of ERF mutations may be lower than previously suggested $(2-3 \%),{ }^{11}$ but analysis of larger cohorts are required to determine this further.

The clinical diagnosis of these syndromes is often difficult, as there are a wide range of overlapping clinical characteristics. The genetic screening cascade analysis is therefore a useful confirmatory tool. In this study, the genetic screening was undertaken by traditional screening methods. Although minor adjustments of our levels 1 and 2 are recommended, such as MLPA screening should be included in level 1 for SCS patients and TCF12 testing in level 2, we recommend offering levels 1 and 2 at the routine diagnostic level (Supplementary Figure 4). However, as NGS costs decrease, this will substitute the traditional approach. In the 65 patients with no genetic mutation detected, exome or genome sequencing will be employed for the identification of mutations in alternative or novel genes implicated in craniosynostosis.

\section{CONFLICT OF INTEREST}

The authors declare no conflict of interest.

\section{ACKNOWLEDGEMENTS}

This work was supported in part by the Ministerio de Innovación y Ciencia (MINECO SAF2012-30871) and ENDOSCREEN (Comunidad de Madrid, S2010/BMD-2396). We wish to thank Noelia Sánchez, Nacho Arriaga and Carolina de la Torre for their technical support; and to Rocio Mena and Inmaculada Rueda for the DNA sequencing.

1 Boulet SL, Rasmussen SA, Honein MA. A population-based study of craniosynostosis in metropolitan Atlanta, 1989-2003. Am J Med Genet 2008; 146A: 984-991.

2 Wilkie AO, Byren JC, Hurst JA et al: Prevalence and complications of single-gene and chromosomal disorders in craniosynostosis. Pediatrics 2010; 126: e391-e400.

3 Muenke M, Gripp KW, McDonald-McGinn DM et al: A unique point mutation in the fibroblast growth factor receptor 3 gene (FGFR3) defines a new craniosynostosis síndrome. Am J Hum Genet 1997; 60: 555-564.

4 Poliakov A, Cotrina M, Wilkinson DG. Diverse roles of eph receptors and ephrins in the regulation of cell migration and tissue assembly. Dev Cell 2004; 7: 465-480.

5 Twigg SR, Kan R, Babbs $\mathrm{C}$ et al: Mutations of ephrin-B1 (EFNB1), a marker of tissue boundary formation, cause craniofrontonasal syndrome. Proc Natl Acad Sci USA 2004; 101: 8652-8657.

6 Twigg SR, Matsumoto K, Kidd AM et al: The origin of EFNB1 mutations in craniofrontonasal syndrome: frequent somatic mosaicism and explanation of the paucity of carrier males. Am J Hum Genet 2006; 78: 999-1010. 
7 Twigg SR, Babbs C, van den Elzen ME et al: Cellular interference in craniofrontonasa syndrome: males mosaic for mutations in the X-linked EFNB1 gene are more severely affected than true hemizygotes. Hum Mol Genet 2013; 22: 1654-1662.

8 Morriss-Kay GM, Wilkie AO. Growth of the normal skull vault and its alteration in craniosynostosis: insights form human genetics and experimental studies. J Anat 2005 207: 637-653.

9 Sharma VP, Fenwick AL, Brockop MS et al: Mutations in TCF12, encoding a basic helix-loop-helix partner of TWIST1, are a frequent cause of coronal craniosynostosis. Nat Genet 2013; 45: 304-307.

10 Le Tanno P, Poreau B, Devillard F et al: Maternal complex chromosomal rearrangement leads to TCF12 microdeletion in a patient presenting with coronal craniosynostosis and intellectual disability. Am J Med Genet 2014; 9999: 1-7.

11 Twigg SR, Vorgia E, McGowan SJ et al: Reduced dosage of ERF causes complex craniosynostosis in humans and mice and links ERK1/2 signaling to regulation of osteogenesis. Nat Genet 2013; 45: 308-313.

12 Woods RH, Ul-Haq E, Wilkie AO et al: Reoperation for intracranial hypertension in TWIST1-confirmed Saethre-Chotzen syndrome: a 15-year review. Plast Reconstr Surg 2009; 123: 1801-1810.

13 Roscioli T, Elakis G, Cox TC et al Genotype and clinical care correlations in craniosynostosis: findings from a cohort of 630 Australian and New Zealand patients. Am J Med Genet C Semin Med Genet 2013; 163C: 259-270.

14 Kress W, Schropp C, Lieb G et al: Saethre-Chotzen syndrome caused by TWIST 1 gene mutations: functional differentiation from Muenke coronal synostosis syndrome. Eur J Hum Genet 2006; 14: 39-48.

15 Mansour SL, Twigg SRF, Freeland RM, Wall SA, Li C, Wilkie AOM. Hearing loss in a mouse model of Muenke syndrome. Hum Mol Genet 2009; 18: 43-50.

16 Ingersoll RG, Paznekas WA, Tran AK, Scott AF, Jiang G, Jabs EW. Fibroblast growth factor receptor 2 (FGFR2): genomic sequence and variations. Cytogenet Cell Genet 2001; 94: 121-126.

17 Desviat LR, Pérez B, Ugarte M. Minigenes to confirm exon skipping mutations. Methods Mol Biol 2012; 867: 37-47.
18 Elanko N, Sibbring JS, Metcalfe KA et al: A survey of TWIST for mutations in craniosynostosis reveals a variable length polyglycine tract in asymptomatic individuals. Hum Mutat 2001; 18: 535-541.

19 Barge-Schaapveld DQCM Lequin MH, van Spaendonk R, Vermeulen RJ, Cobben JM. Beare-Stevenson syndrome: two Dutch patients with cerebral abnormalities. Pediatr Neurol 2011; 44: 303-307.

20 Akai T, lizuka H, Kishibe M, Kawakami S, Kobayashi A, Ozawa T. A case of Beare-Stevenson cutis gyrate syndrome confirmed by mutation analysis of the fibroblast growth factor receptor 2 gene. Pediatr Neurosurg 2002; 37: 97-99.

21 Arnaud-López L, Fragoso R, Mantilla-Capacho J, Barros-Núñez P. Crouzon with acanthosis nigricans. Further delineation of the syndrome. Clin Genet 2007; 72: 405-410.

22 Oldridge M, Lunt PW, Zackai EH et al: Genotype-phenotype correlation for nucleotide substitutions in the IgII-IgIII linker of FGFR2. Hum Mol Genet 1997; 6: 137-143.

23 Lajeunie E, Cameron R, El Ghouzzi V et al: Clinical variability in patients with Apert's syndrome. J Neurosurg 1999; 90: 443-447.

24 Oldridge M, Zackai EH, McDonald-McGinn DM et al: De novo alu-element insertions in FGFR2 identify a distinct pathological basis for Apert syndrome. Am J Hum Genet 1999; 64: 446-461.

25 Bochukova EG, Roscioli T, Hedges DJ et al: Rare mutations of FGFR2 causing apert syndrome: identification of the first partial gene deletion, and an Alu element insertion from a new subfamily. Hum Mutat 2009; 30: 204-211.

26 Fenwick AL, Bowdin SC, Klatt REM, Wilkie AOM. A deletion of FGFR2 creating a chimeric IIIb/IIlc exon in a child with Apert syndrome. BMC Med Genet 2011; 12: 122 .

27 Wilkie AO, Bochukova EG, Hansen RM et al: Clinical dividends from the molecula genetic diagnosis of craniosynostosis. Am J Med Genet 2007; 143A: 1941-1949.

28 Klopocki E, Lohan S, Brancati F. Copy-number variations involving the IHH locus are associated with syndactyly and craniosynostosis. Am J Hum Genet 2011; 88: 70-75.

29 Barroso E, Pérez-Carrizosa V, García-Recuero I et al: Mild isolated craniosynostosis due to a novel FGFR3 mutation. Am J Med Genet 2011; 155A: A334T-3053.

Supplementary Information accompanies this paper on European Journal of Human Genetics website (http://www.nature.com/ejhg) 